Swarthmore College

Works

$12-31-2013$

\title{
Planar Anchoring Strength And Pitch Measurements In Achiral And Chiral Chromonic Liquid Crystals Using 90-Degree Twist Cells
}

Christine K. McGinn , '16

Laura I. Laderman , '15

N. Zimmermann

H.-S. Kitzerow

Peter J. Collings

Swarthmore College, pcollin1@swarthmore.edu

Follow this and additional works at: https://works.swarthmore.edu/fac-physics

Part of the Physics Commons

Let us know how access to these works benefits you

\section{Recommended Citation}

Christine K. McGinn , '16; Laura I. Laderman , '15; N. Zimmermann; H.-S. Kitzerow; and Peter J. Collings. (2013). "Planar Anchoring Strength And Pitch Measurements In Achiral And Chiral Chromonic Liquid Crystals Using 90-Degree Twist Cells". Physical Review E. Volume 88, Issue 6. DOI: 10.1103/

PhysRevE.88.062513

https://works.swarthmore.edu/fac-physics/126

This work is brought to you for free by Swarthmore College Libraries' Works. It has been accepted for inclusion in Physics \& Astronomy Faculty Works by an authorized administrator of Works. For more information, please contact myworks@swarthmore.edu. 


\title{
Planar anchoring strength and pitch measurements in achiral and chiral chromonic liquid crystals using 90-degree twist cells
}

\author{
Christine K. McGinn, ${ }^{1}$ Laura I. Laderman, ${ }^{1}$ Natalie Zimmermann, ${ }^{2}$ Heinz-S. Kitzerow, ${ }^{2}$ and Peter J. Collings ${ }^{1,3}$ \\ ${ }^{1}$ Department of Physics \& Astronomy, Swarthmore College, Swarthmore, Pennsylvania 19081, USA \\ ${ }^{2}$ Department of Chemistry, University of Paderborn, 33098 Paderborn, F. R. Germany \\ ${ }^{3}$ Department of Physics and Astronomy, University of Pennsylvania, Philadelphia, Pennsylvania 19104, USA
}

(Received 21 September 2013; published 31 December 2013)

\begin{abstract}
Chromonic liquid crystals are formed by molecules that spontaneously assemble into anisotropic structures in water. The ordering unit is therefore a molecular assembly instead of a molecule as in thermotropic liquid crystals. Although it has been known for a long time that certain dyes, drugs, and nucleic acids form chromonic liquid crystals, only recently has enough knowledge been gained on how to control their alignment so that studies of their fundamental liquid crystal properties can be performed. In this article, a simple method for producing planar alignment of the nematic phase in chromonic liquid crystals is described, and this in turn is used to create twisted nematic structures of both achiral and chiral chromonic liquid crystals. The optics of 90-degree twist cells allows the anchoring strength to be measured in achiral systems, which for this alignment technique is quite weak, about $3 \times 10^{-7} \mathrm{~J} / \mathrm{m}^{2}$ for both disodium cromoglycate and Sunset Yellow FCF. The addition of a chiral amino acid to the system causes the chiral nematic phase to form, and similar optical measurements in 90-degree twist cells produce a measurement of the intrinsic pitch of the chiral nematic phase. From these measurements, the helical twisting power for $L$-alanine is found to be $(1.1 \pm 0.4) \times 10^{-2} \mu \mathrm{m}^{-1} \mathrm{wt} \%^{-1}$ for $15 \mathrm{wt} \%$ disodium cromoglycate.
\end{abstract}

DOI: 10.1103/PhysRevE.88.062513

PACS number(s): 61.30.Gd, 61.30.Hn, 78.15.+e

\section{INTRODUCTION}

The materials used in liquid crystal displays (LCDs) are called thermotropic liquid crystals since they are composed of a pure compound or mixture of compounds in which the anisotropic molecules spontaneously order orientationally and sometimes positionally within a certain temperature range. Thus phase changes from solid to liquid crystal and from liquid crystal to isotropic liquid are produced by varying the temperature. These materials have been studied for both scientific and applied reasons, and the ubiquitous role of LCDs in today's world is due to the huge amount of understanding and control of these materials that has been gained over the last half century. There are other types of liquid crystals that form when compounds are added to a solvent and the structures that spontaneously form order into a liquid crystal phase. These materials are called lyotropic liquid crystals because phase changes can result by varying the concentration of the compound. One example of a lyotropic liquid crystal is the phase formed by certain dyes, drugs, and nucleic acids. When added to water these compounds spontaneously assemble into stacks of molecules, creating rod-like structures that are anisotropic. These materials are called chromonic liquid crystals, and at certain concentrations and temperatures, these assemblies form either the most simple liquid crystal phase with orientational order only (the nematic phase) or a more complex liquid crystal phase in which there is some degree of positional ordering of the oriented assemblies (the columnar phase). From a macroscopic standpoint, the nematic phases of thermotropic and chromonic liquid crystals are identical $[1,2]$.

The existence of chromonic liquid crystals has been known for over fifty years, with most of the studies during this period concentrating on $x$-ray measurements of the phase structures and determinations of temperature-concentration phase diagrams. Recently the amount of interest in these materials has grown significantly, mostly due to the fact that these are aqueous systems (unlike thermotropic liquid crystals), so applications in biology and medicine are anticipated [3]. In fact, it has already been shown that chromonic liquid crystals can be used to detect the presence of toxins in a rather simple device [4,5]. This high level of recent interest has begun to probe the fundamental liquid crystalline properties of these materials, producing not only measurements of various parameters, but also allowing for control of the liquid crystal alignment direction. Examples of such studies include measurements of the order parameter, birefringence, elastic constants, and polar anchoring strength [6-10]. Almost all of these measurements have been in achiral systems, with only a few reports of studies done on chiral nematic chromonic liquid crystals $[3,11,12]$.

Planar alignment of the nematic phase of chromonic liquid crystals has been achieved by polarized photo-irradiation of an azo-polymer thin film $[13,14]$, rubbing a polymer coating [7,15], evaporating a $\mathrm{SiO}_{X}$ layer [15], and fabricating a surface of closely spaced ridges as in Refs. $[16,17]$. The effectiveness of these techniques varies considerably from one material to another, and some involve multiple, sophisticated fabrication steps. It turns out that it is possible to produce excellent alignment of the nematic phase in chromonic liquid crystals by simply rubbing glass with a very fine abrasive [10]. This very simple but effective technique then allows a wide range of experiments to be performed. For example, if the chromonic liquid crystal is placed between two flat pieces of glass with rubbing directions at $90^{\circ}$ to each other, the liquid crystal adopts a twisted structure that results from a competition between volume twist elastic energy and surface anchoring energy. Measuring the amount of twist in such a 90-degree twist cell allows the planar anchoring strength to be measured since the elastic constants are already 
known. This use of a twist cell to measure planar anchoring strength has been used in the past with a thermotropic liquid crystal [18]. The results presented here are an anchoring strength of $(3.4 \pm 0.6) \times 10^{-7} \mathrm{~J} / \mathrm{m}^{2}$ for disodium dicromoglycate (DSCG) and $(3.3 \pm 0.9) \times 10^{-7} \mathrm{~J} / \mathrm{m}^{2}$ for Sunset Yellow FCF (SSY). When compared to the anchoring strength achieved by the many commercial methods to align thermotropic liquid crystals, this anchoring strength is quite weak [19]. This is not surprising given that the alignment mechanism involves closely spaced parallel scratches in glass. For fabricated ridges of optimized separation and height, the anchoring strength for chromonic liquid crystals has been reported to be much larger [17].

It is known that addition of a water soluble chiral compound causes the chromonic liquid crystal orientation to twist in what is known as the chiral nematic phase $[3,11,12]$. Understanding how these chiral nematics orient in a 90-degree twist cell allows the intrinsic pitch of the chiral nematic phase to be measured from optical measurements because the anchoring strength is known. Determining how the pitch depends on the concentration of the chiral compound allows the helical twisting power to be measured for quite large pitch lengths, the accuracy of which can be verified by producing fingerprint textures for samples with smaller pitches.

The compounds used in this investigation are shown in Fig. 1. DSCG and SSY are chromonic liquid crystals, whereas<smiles></smiles>

Disodium Cromoglycate (DSCG)

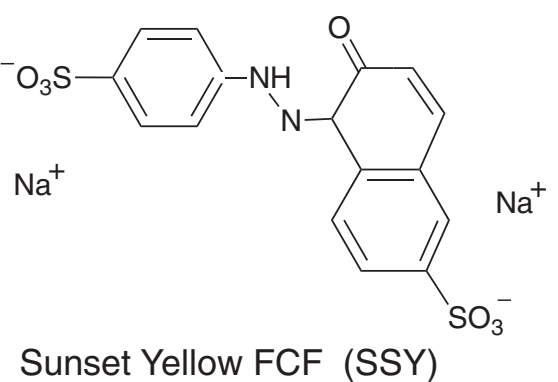<smiles>C[C](N)C(=O)O</smiles>

L-Alanine

FIG. 1. Compounds used in this investigation.

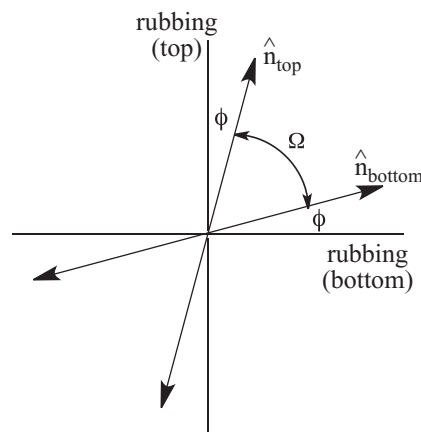

(a) right-handed twist domain

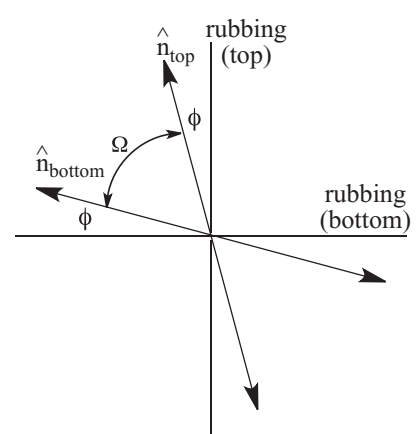

(b) left-handed twist domain
FIG. 2. Right- and left-handed twist domains. The substrate alignment directions are perpendicular, the director orientation at the substrates is offset by an angle $\phi$ from the alignment direction, and the director twists through an angle $\Omega$ between the two substrates.

$L$-alanine is the chiral dopant. DSCG, SSY, and $L$-alanine were purchased from Sigma-Aldrich.

\section{THEORETICAL CONSIDERATIONS}

\section{A. Elastic theory}

If a nematic liquid crystal is placed between two substrates promoting planar alignment, but the two alignment directions are perpendicular to each other, then the director usually assumes a structure with only twist deformation [20]. This is especially true for chromonic liquid crystals for which twist deformation involves less elastic free energy than splay or bend deformation [8,10]. Also, since the amount of twist deformation can be reduced if the director next to each substrate is not exactly aligned with the alignment direction of the substrate, there is an offset angle $\phi$ at each substrate. If the angle by which the director twists is denoted by $\Omega$, then $\Omega+2 \phi=\pi / 2$. Figure 2 depicts the situation for both rightand left-handed twist domains. Clearly the values of $\Omega$ and $\phi$ depend on the elastic and anchoring parameters along with the distance between the substrates, $d$.

The free energy associated with distortion of the director of a chiral nematic liquid crystal is given by the Frank free energy, with the energy per unit volume $f_{V}$ given by

$$
\begin{aligned}
f_{V}= & -k_{2}(\hat{n} \cdot \nabla \times \hat{n})+\frac{1}{2} k_{11}(\nabla \cdot \hat{n})^{2}+\frac{1}{2} k_{22}(\hat{n} \cdot \nabla \times \hat{n})^{2} \\
& +\frac{1}{2} k_{33}|\hat{n} \times(\nabla \times \hat{n})|^{2}
\end{aligned}
$$

where $\hat{n}$ is the director, $k_{11}, k_{22}$, and $k_{33}$ are the splay, twist, and bend elastic constants, respectively, and $k_{2}$ is the parameter related to the intrinsic chirality of the liquid crystal [20]. The intrinsic chirality of the liquid crystal is given by $q_{0}$, which is equal to $k_{2} / k_{22}$. The pitch $P$ is related to the intrinsic chirality by $q_{0}=2 \pi / P$. The free energy per unit area associated with the offset of the director from the substrate alignment direction $f_{S}$ is given in its most simple form by the Rapini expression,

$$
f_{S}=\frac{1}{2} W_{\phi} \sin ^{2} \phi
$$




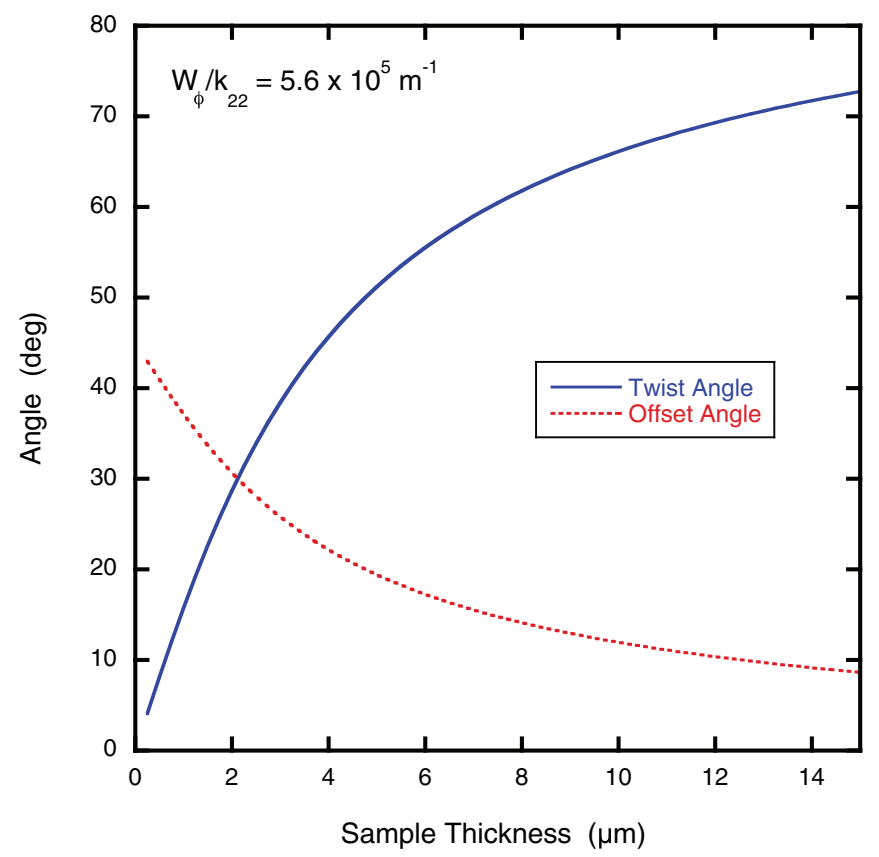

FIG. 3. (Color online) Theoretical variation of the twist and offset angles as a function of sample thickness with $W_{\phi} / k_{22}=5.6 \times$ $10^{5} \mathrm{~m}^{-1}$.

where $W_{\phi}$ is the anchoring strength and $\phi$ is the angle between the alignment direction of the substrate and the director at the substrate [20]. For pure twist distortion between two substrates a distance $d$ apart, the total free energy per unit volume is given by

$$
\begin{aligned}
f_{V}+f_{S} / d+f_{S} / d= & -k_{2}(\Omega / d)+\frac{1}{2} k_{22}(\Omega / d)^{2} \\
& +\frac{1}{2}\left(W_{\phi} / d\right) \sin ^{2} \phi+\frac{1}{2}\left(W_{\phi} / d\right) \sin ^{2} \phi,
\end{aligned}
$$

which is minimized when

$$
\frac{W_{\phi} d}{2 k_{22}} \cos \Omega-\Omega+q_{0} d=0 \quad \text { or } a \cos \Omega-\Omega+q_{0} d=0 \text {, }
$$

where $a$ equals $W_{\phi} d /\left(2 k_{22}\right)$.

Let us first examine the case of an achiral nematic phase for which $q_{0}=0$. If $d$ is small enough, $a \cos \Omega \ll \Omega$, so $\Omega \rightarrow 0$ and $\phi \rightarrow \pi / 4$. This means the liquid crystal is undistorted, pointing at an angle of $45^{\circ}$ relative to each of the substrate alignment directions. If $d$ is large enough, $a \cos \Omega$ can only equal $\Omega$ if $\Omega \rightarrow \pi / 2$ and $\phi \rightarrow 0$. This means that the twist is $90^{\circ}$ and the director at each substrate is parallel to the substrate alignment direction. A plot showing the dependence of the twist and offsets angles on the distance between the substrates using parameters that correspond to the results presented here is shown in Fig. 3.

The case of a chiral nematic liquid crystal with nonzero $q_{0}$ is a bit more complicated. For low intrinsic chirality (large pitch), the twist angle $\Omega$ is increased and the offset angle $\phi$ is decreased because the liquid crystal prefers to be twisted. As the intrinsic chirality increases, however, first $\Omega$ increases past $\pi / 2$ with $\phi$ taking on negative values (i.e., the director at the

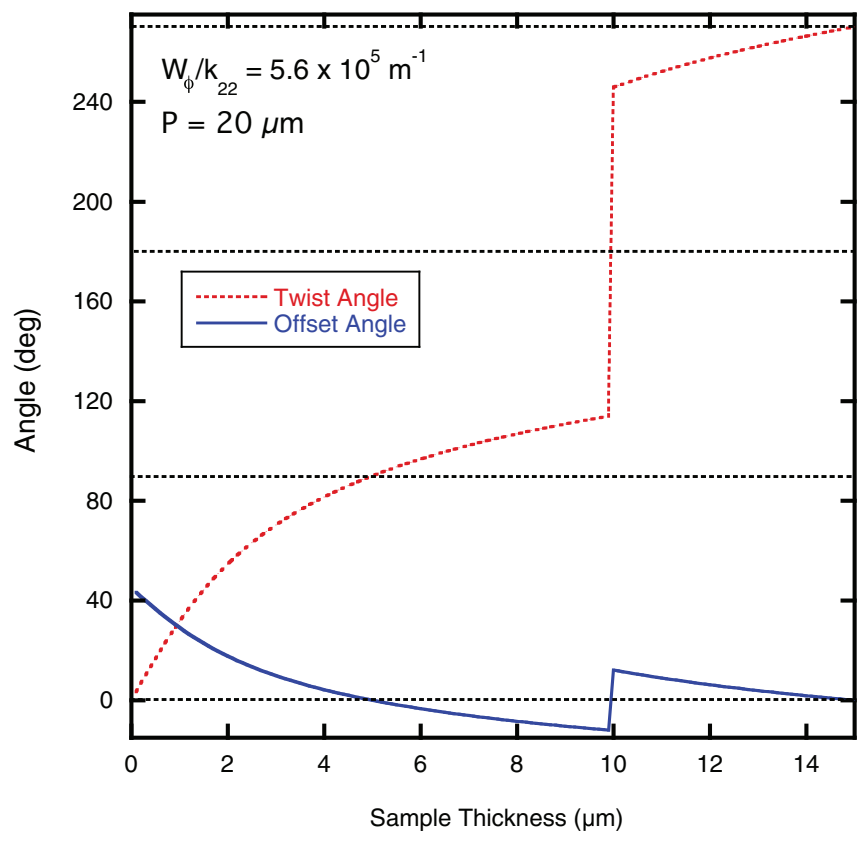

FIG. 4. (Color online) Theoretical variation of the twist and offset angles as a function of sample thickness with $W_{\phi} / k_{22}=5.6 \times$ $10^{5} \mathrm{~m}^{-1}$ and $P=20 \mu \mathrm{m}$. Notice that when the sample thickness is $5 \mu \mathrm{m}(P / 4)$, the twist angle is exactly $90^{\circ}$ and the offset angle is zero. When the sample thickness is $10 \mu \mathrm{m}$, the most stable twist angle jumps from $114^{\circ}$ to $246^{\circ}$ and the offset angle jumps from $-12^{\circ}$ to $+12^{\circ}$. Finally, when the sample thickness is $15 \mu \mathrm{m}(3 P / 4)$, the twist angle is exactly $270^{\circ}$ and the offset angle is again zero.

substrate is on the other side of the alignment direction from what is shown in Fig. 2), until a lower elastic energy condition exists for a value of $\Omega$ approaching $3 \pi / 2$. This behavior can be seen in a plot of the twist and offset angles versus the distance between the substrates for a material with fixed intrinsic chirality (see Fig. 4). Such behavior is well known for thermotropic liquid crystals, both for parallel substrate rubbing (Grandjean-Cano wedge) and nonparallel substrate rubbing [21].

\section{B. Optical theory}

The transmission of light through a twisted nematic cell was the focus of research in the early days of liquid crystal displays. For example, an exact calculation using coupled mode theory was worked out by McIntyre in 1978 [22]. One of the important considerations in understanding the optics of twisted nematic cells is the relationship between the twist angle $\Omega$ and the amount of phase retardation $\psi=(2 \pi / \lambda) \Delta n d$, where $\lambda$ is the vacuum wavelength of the light and $\Delta n$ is the birefringence of the liquid crystal. If $|\Omega| \ll|\psi|$, then the Mauguin or adiabatic condition is met and the polarization direction of linearly polarized incident light simply follows the twist of the director. If this condition is not met, then even if the incident light is linearly polarized, (1) the transmitted light is no longer linearly polarized, and (2) the major axis of the transmitted elliptically polarized light is not rotated by the twist angle $\Omega$. For chromonic liquid crystals $(\Delta n \approx-0.02)$ in a 10 - $\mu$ m-thick cell with $|\Omega|=(1 / 3) \pi,|\psi|$ is roughly $(4 / 5) \pi$ 
for visible light [7]. While $|\Omega|$ is less than $|\psi|$, the inequality is not large enough to ensure that the Mauguin condition is met. Any analysis of experimental data must therefore use the exact calculation.

Perhaps the most simple way to conduct an experiment with a twisted nematic cell is to use a linear polarizer to polarize the incident monochromatic light and detect only the light that exits the cell and passes through a linear polarizer aligned either perpendicular or parallel to the incident polarizer. The twisted nematic cell can then be rotated around an axis parallel to the light propagation direction and the intensity exiting the second polarizer monitored. The theory of McIntyre predicts that the intensity depends on the rotation angle of the twisted nematic cell $\theta$ according to

$$
I(\theta)=I_{C} \cos ^{2}(2 \theta+\Omega)+I_{S} \sin ^{2}(2 \theta+\Omega),
$$

where $I_{C}$ and $I_{S}$ are fractions of the incident light intensity that depend on $\Omega, \Delta n, d$, and $\lambda$. $I_{C}$ and $I_{S}$ are the maximum and minimum values of $I(\theta)$, although which one is which depends on how $\theta$, the angle between a direction in the twist cell and a direction of the polarizers, is defined and whether the polarizers are perpendicular or parallel. For example, if $X$ is the ratio of half the retardation angle to the twist angle, i.e., $X=\psi /(2 \Omega)$, then for parallel polarizers

$$
\begin{aligned}
I_{C}= & {\left[\cos \tau \cos \Omega+\frac{1}{\sqrt{1+X^{2}}} \sin \tau \sin \Omega\right]^{2} } \\
& +\left[\frac{X}{\sqrt{1+X^{2}}} \sin \tau\right]^{2}, \\
I_{S}= & {\left[\cos \tau \cos \Omega+\frac{1}{\sqrt{1+X^{2}}} \sin \tau \sin \Omega\right]^{2}, }
\end{aligned}
$$

where $\tau=\Omega \sqrt{1+X^{2}}$.

If $d$ and $\lambda$ are known for an achiral liquid crystal, then $\Omega$ and $\Delta n$ can be determined from measurements of $I_{C}$ and $I_{S}$. Once $\Omega$ has been determined, elastic theory can be used to obtain $W_{\phi} / k_{22}$, and if the value of $k_{22}$ is known for the liquid crystal, then the value of $W_{\phi}$ can be found.

It is interesting to investigate how $I_{C}$ and $I_{S}$ depend on $d$ according to elastic and optical theory. When $d$ is very small, the liquid crystal does not change the polarization of the light. Therefore, $I_{C}=I_{S}=0$ when the polarizers are perpendicular, and $I_{C}=I_{S}=1$ when the polarizers are parallel. As $d$ increases, $I_{C}$ and $I_{S}$ increase from zero (one more quickly than the other) for perpendicular polarizers and $I_{C}$ and $I_{S}$ decrease from one (one more quickly than the other) for parallel polarizers. This is illustrated in Fig. 5 .

Assuming $\Delta n$ and $W_{\phi} / k_{22}$ are not changed by the addition of a small amount of a chiral compound, then a similar experiment that determines $I_{C}$ and $I_{S}$ for parallel and perpendicular polarizers can be combined with elastic theory (nonzero $q_{0}$ this time) and optical theory to determine $q_{0}$ and hence the pitch $P$. The dependence of the transmitted light on sample thickness using the same parameters for the liquid crystal but with a nonzero $q_{0}$ is also shown in Fig. 5.

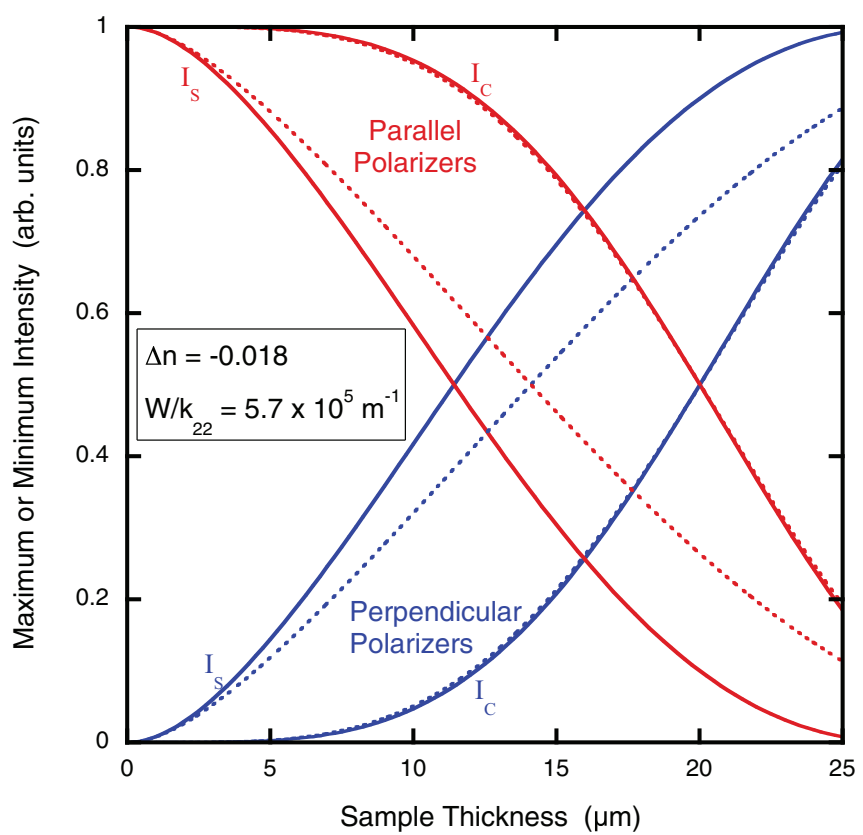

FIG. 5. (Color online) Theoretical maximum and minimum intensities, $I_{C}$ and $I_{S}$, for $633 \mathrm{~nm}$ light versus sample thickness with $W_{\phi} / k_{22}=5.7 \times 10^{5} \mathrm{~m}^{-1}$ and $\Delta n=-0.018$. The intensities with parallel and perpendicular polarizers start at 1 and 0 , respectively, at zero sample thickness. The solid lines are for the nonchiral case and the dotted lines are for a pitch of $60 \mu \mathrm{m}$. The $I_{C}$ and $I_{S}$ labels are for a specific definition of the angle $\theta$ in Eq. (5).

\section{EXPERIMENTAL PROCEDURES}

\section{A. 90-degree twist cells}

DSCG was used without further purification, but SSY was purified twice by dissolving the sample in water, adding ethanol until a good deal of the compound precipitated, filtering, and allowing the filtrate to dry [6]. Mixtures of DSCG at 14.9 and $15.0 \mathrm{wt} \%$ in millipore water were prepared, which had a nematic phase at room temperature and a nematicisotropic biphasic region from 29 to $36{ }^{\circ} \mathrm{C}$ upon heating. A $30 \mathrm{wt} \%$ mixture of SSY was similarly prepared, which had a nematic phase at room temperature and a nematic-isotropic biphasic region from 30 to $43^{\circ} \mathrm{C}$ upon heating. Chiral nematic phases of DSCG were made by first preparing an $L$-alanine solution in millipore water at a specified concentration between 1 and $2.5 \mathrm{wt} \%$, and then making a $15.0 \mathrm{wt} \%$ mixture of DSCG in the $L$-alanine-water solution. Alignment of the nematic director on glass was achieved by rubbing the glass once with a fine abrasive pad (3M Trizact ${ }^{\mathrm{TM}}$ Foam Disc P3000) under a pressure of $1.6 \times 10^{4} \mathrm{~N} / \mathrm{m}^{2}$. The glass was then cleaned by overnight soaking in concentrated sulphuric acid or plasma cleaning. When DSCG or SSY is introduced into a $10 \mu \mathrm{m}$ cell with parallel rubbing directions on the glass substrates, a single oriented domain is produced. When the rubbing directions on the glass substrates are perpendicular to one another, domains of right- and left-handed twist form as shown in Fig. 6.

Unlike thermotropic liquid crystals, these domains remain unchanged for hours, i.e., there is almost no evidence of annealing. In fact, if the rubbing directions are oriented so that the angle between them is slightly less than $90^{\circ}$, twist domains 


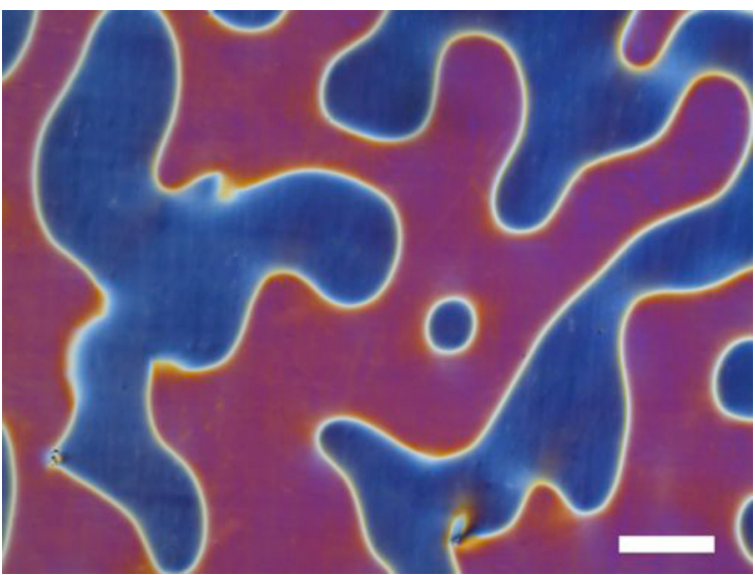

FIG. 6. (Color online) Right- and left-handed twist domains in $15 \mathrm{wt} \%$ DSCG using white light, a $5 \times$ objective, parallel polarizers, and a $\lambda$ plate. The thickness of the sample is roughly $10 \mu \mathrm{m}$. The length of the scale bar corresponds to $300 \mu \mathrm{m}$.

of both handedness still form. Thus an angle of $90^{\circ}$ between the rubbing directions was used in these experiments for two reasons: (1) this is the maximum angle possible and therefore optimizes the competition between the bulk and surface elastic energies, and (2) the twist domains achieved by slow cooling into the nematic phase were large enough for measurements under the microscope.

A wedge cell was prepared using two glass substrates with perpendicular rubbing directions $\left(90^{\circ} \pm 2^{\circ}\right)$ by placing no spacers at one end of a 2.5 -cm-long substrate and 10- or $15-\mu \mathrm{m}$ glass fiber spacers at the other end. These two ends were epoxied to a second glass substrate and the other two sides were left open. This empty wedge cell was placed in a uv-vis spectrophotometer next to an opaque strip with an open slit $125 \mu \mathrm{m}$ wide. The series of maxima and minima in absorbance as the wavelength is scanned is due to interference within the empty cell and allows the thickness of the cell to be measured at the location of the $125 \mu \mathrm{m}$ slit. By placing the $125 \mu \mathrm{m}$ slit at multiple locations along the wedge cell, it was found that the thickness of the cell was nearly linear with position along the cell although some curvature was sometimes present. These data were used to find the thickness at intermediate locations of the cell. The wedge cell was then filled with the DSCG or SSY solution at a temperature in the isotropic phase, sealed completely with epoxy, and allowed to cool to room temperature.

The wedge cell was placed in an Instec HS-1 hot stage at $25{ }^{\circ} \mathrm{C}$ and viewed with a Leitz Laborlux 12 Pol microscope with an objective magnification of $10 \times$ for cells with leftand right-handed twist domains and $4 \times$ for cells with only one twist domain. A $633 \mathrm{~nm}$ laser line filter was used to produce quasimonochromatic light. For each of the positions in the wedge cell for which the thickness was measured or interpolated, a location within a single twist domain (if present) was placed at the center of rotation of the microscope stage. Then the microscope stage was rotated through $90^{\circ}$ in steps of either $5^{\circ}$ or $10^{\circ}$ with the polarizers perpendicular to each other. An image was captured at each step with a Zeiss AxioCam ICc 1 digital camera and the entire procedure was repeated with the polarizers parallel to each other. The images were analyzed by measuring the average intensity within

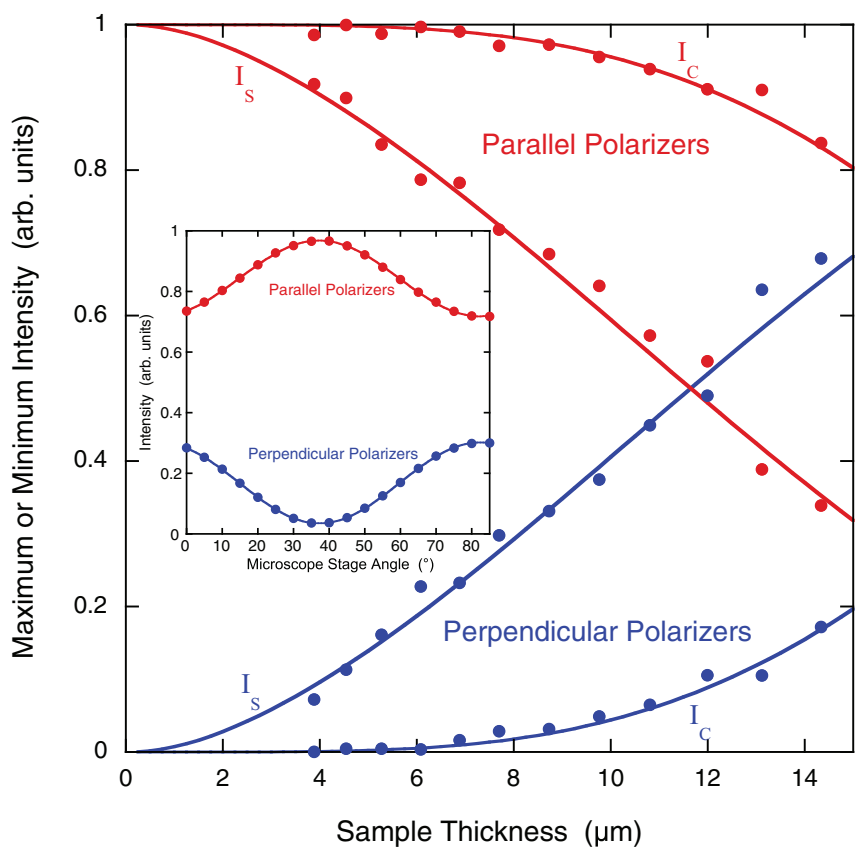

FIG. 7. (Color online) Maximum and minimum intensities as a function of sample thickness for $15 \mathrm{wt} \%$ DSCG at $25^{\circ} \mathrm{C}$. The intensities with parallel and perpendicular polarizers start at 1 and 0 , respectively, at zero sample thickness. The experimental data are shown as solid circles and the solid lines are fits to the elastic and optical theory as described in the text, yielding best-fit values for $\Delta n$ of -0.0177 and $W_{\phi} / k_{22}$ of $5.70 \times 10^{5} \mathrm{~m}^{-1}$, with a determination of $W_{\phi}$ of $(3.4 \pm 0.6) \times 10^{-7} \mathrm{~J} / \mathrm{m}^{2}$. Inset: intensity as a function of microscope stage angle for a $15 \mathrm{wt} \%$ DSCG sample of thickness $7.7 \mu \mathrm{m}$ at $25^{\circ} \mathrm{C}$. The fits are to Eq. (5) after $37.3^{\circ}$ has been subtracted from each angle value.

a circle $74 \mu \mathrm{m}$ in diameter $(10 \times$ objective $)$ or $185 \mu \mathrm{m}$ in diameter $(4 \times$ objective) at the center of the microscope stage rotation using Zeiss ZEN 2011 software. A typical plot of how the intensity varied with angle for both polarizer conditions is shown in the inset of Fig. 7.

\section{B. Fingerprint textures}

Fingerprint textures have been observed for chromonic liquid crystals, but they are not nearly as robust as for thermotropic liquid crystals $[3,11,12]$. The best one can do seems to be a texture with some areas showing the half-pitch repeat distance because the helical pitch axis is parallel to the glass surfaces, but with many areas in between showing no repeating pattern. In addition, when measuring the repeat distance under a microscope, large variations in the spacing of the repeating pattern are seen from one area to another. It is known that coating the glass surface with the polymer parylene tends to produce a homeotropic texture for SSY [23]. Using this technique in 50- or 100- $\mu \mathrm{m}$-thick cells did produce a fingerprint texture in some areas for both DSCG and SSY samples if $L$-alanine is added, and a few concentrations of $L$-alanine in $15 \mathrm{wt} \%$ DSCG were found to give reasonable estimates of the pitch as a check on the measurements made with the twist cells. 


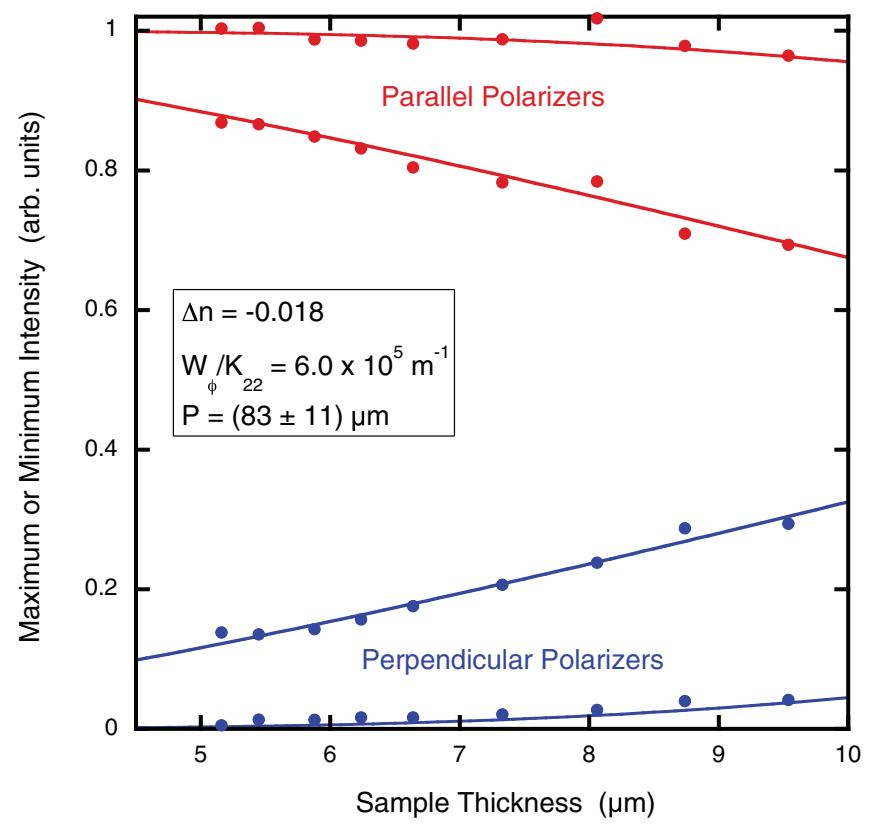

FIG. 8. (Color online) Maximum and minimum intensities as a function of sample thickness for $15 \mathrm{wt} \%$ DSCG mixed with a $1 \mathrm{wt} \%$ $L$-alanine solution at $25^{\circ} \mathrm{C}$. The experimental data are shown as solid circles and the solid lines are fits to the elastic and optical theory as described in the text and yield a value for the pitch of $(83 \pm 11) \mu \mathrm{m}$.

\section{EXPERIMENTAL RESULTS}

The intensity readings as the microscope stage was rotated were normalized by first subtracting the minimum reading for perpendicular polarizers with the thinnest sample thickness and second dividing the result by the maximum reading for parallel polarizers with the thinnest sample thickness. Values of $I_{C}$ and $I_{S}$ were then determined by fitting the data to Eq. (5) as shown in the inset of Fig. 7. A typical set of intensity readings at various sample thicknesses in a wedge cell together with the fits to the data using elastic and optical theory are shown for both an achiral and a chiral sample in Figs. 7 and 8, respectively. The results of this procedure for the achiral solution were $\Delta n=-0.0177$ (for $633 \mathrm{~nm}$ light) and $W_{\phi} / k_{22}=5.70 \times 10^{5} \mathrm{~m}^{-1}$. This value of $\Delta n$ is consistent with prior measurements on DSCG [7]. It should be pointed out that the fit yields a twist angle $\Omega$ ranging from $40^{\circ}$ to $70^{\circ}$ and an offset angle $\phi$ ranging from $25^{\circ}$ to $10^{\circ}$ for the sample thicknesses investigated. The elastic constant $k_{22}$ has been measured for DSCG and for a $14.9 \mathrm{wt} \%$ mixture at $25^{\circ}$ is about $0.6 \times 10^{-12} \mathrm{~N}$ [8]. This yields a value for the anchoring strength $W_{\phi}$ of $(3.4 \pm 0.6) \times 10^{-7} \mathrm{~J} / \mathrm{m}^{2}$, where the error estimate reflects the uncertainty in $W_{\phi} / k_{22}$ only. Since the chiral samples were made with a slightly higher DSCG concentration, the values of the birefringence were kept between -0.017 and -0.020 and $W_{\phi} / k_{22}$ was fixed at $6.0 \times 10^{5} \mathrm{~m}^{-1}$ when fitting the data to determine the pitch for the chiral samples.

The birefringence at $633 \mathrm{~nm}$ is significantly higher for SSY than for DSCG. As the birefringence increases in a twist domain, $I_{C}$ and $I_{S}$ "oscillate" between values near 0 and 1 (see Fig. 5). This means that for larger sample thicknesses

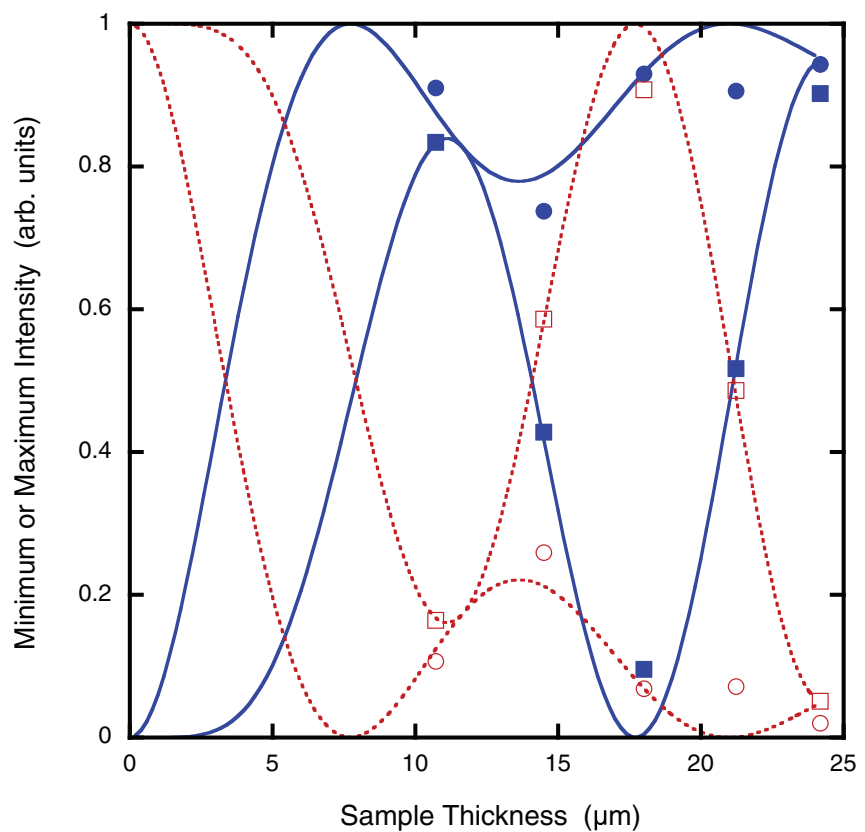

FIG. 9. (Color online) Maximum and minimum intensities as a function of sample thickness for a $30 \mathrm{wt} \%$ solution of Sunset Yellow $\mathrm{FCF}$ at $26^{\circ} \mathrm{C}$. The minimum and maximum intensities with parallel polarizers are the red open circles and open squares, respectively, and the fits are the dotted lines. The minimum and maximum intensities with perpendicular polarizers are the blue solid squares and solid circles, respectively, and the fits are solid lines. The fits are to the elastic and optical theory as described in the text and yield bestfit values for $\Delta n$ of -0.051 and $W_{\phi} / k_{22}$ of $4.7 \times 10^{5} \mathrm{~m}^{-1}$, with a determination of $W_{\phi}$ of $(3.3 \pm 0.9) \times 10^{-7} \mathrm{~J} / \mathrm{m}^{2}$.

of SSY, $I_{C}$ and $I_{S}$ have gone through one or more of these "oscillations." In an attempt to follow this evolution with sample thickness, thin (3-4 $\mu \mathrm{m})$, standard (5-12 $\mu \mathrm{m})$, and thick $(11-24 \mu \mathrm{m})$ wedge cells were made. It was found that $I_{C}$ and $I_{S}$ do oscillate as expected, but the thinner the sample thickness, the less the data were in accordance with the theory. So in order to get a determination of $\Delta n$ and $W_{\phi} / k_{22}$, only data from the thick wedge cell were analyzed completely. This is shown in Fig. 9, where it is clear that the data for DSCG are much better. The best fit to the data results in a $\Delta n$ of 0.051 and a $W_{\phi} / k_{22}$ of $4.7 \times 10^{5} \mathrm{~m}^{-1}$. This means that in going from a sample thickness of 11 to $24 \mu \mathrm{m}$, the twist angle $\Omega$ ranges from $64^{\circ}$ to $76^{\circ}$ and the offset angle $\phi$ ranges from $13^{\circ}$ to $7^{\circ}$.

The birefringence of SSY has been measured for solutions in the same concentration range and with the same transition temperatures $[6,10]$. The value is between -0.06 and -0.07 , which is higher in magnitude than the result of the fit from these data. The value of the parameter $k_{22}$ has also been measured and is about $0.7 \times 10^{-12} \mathrm{~N}$ for this range of concentrations and transition temperatures [10]. Using this value, the determination for the anchoring strength of SSY is $(3.3 \pm 0.9) \times 10^{-7} \mathrm{~J} / \mathrm{m}^{2}$, where the error estimate includes the uncertainties in $W_{\phi} / k_{22}$ and $k_{22}$. This value is very similar to the result for DSCG.

The pitch measurements for $15 \mathrm{wt} \%$ DSCG in various concentrations of $L$-alanine and water can be presented by plotting the inverse pitch versus $L$-alanine concentration. The 


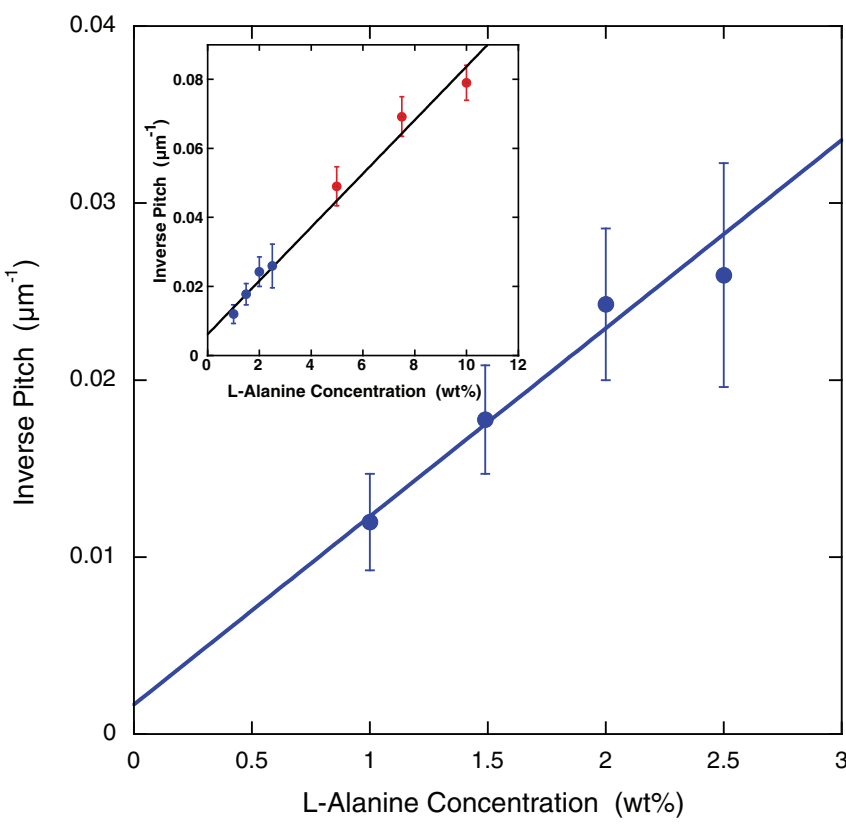

FIG. 10. (Color online) The dependence of the inverse pitch on the $L$-alanine concentration for $15 \mathrm{wt} \%$ DSCG using the $90^{\circ}$ twist cell technique. The helical twisting power is $(1.1 \pm 0.4) \times$ $10^{-2} \mu \mathrm{m}^{-1} \mathrm{wt} \%^{-1}$ and the inverse pitch intercept is $(0.002 \pm$ 0.006) $\mu \mathrm{m}^{-1}$. The inset shows these same data along with three measurements at higher concentrations of $L$-alanine (shown in red) using fingerprint textures.

data should show a trend that is consistent with zero inverse pitch at zero $L$-alanine concentration and the slope is called the helical twisting power of $L$-alanine in this liquid crystal. The pitch measurements obtained from the twist cells are plotted in this way in Fig. 10, where a linear fit to the data yields a helical twisting power $(\mathrm{HTP})$ of $(1.1 \pm 0.4) \times 10^{-2} \mu \mathrm{m}^{-1} \mathrm{wt} \%{ }^{-1}$ and the fit is consistent with zero inverse pitch at zero concentration of $L$-alanine. As a check, the inset to Fig. 10 shows that the pitch measurements from the twist cells and the fingerprint textures are consistent with one another.

In an attempt to see if the change from a twist angle of slightly less than $120^{\circ}$ to slightly more than $240^{\circ}$ predicted theoretically in Fig. 4 might be observed, a wedge twist cell was prepared and filled with $15 \mathrm{wt} \%$ DSCG in a $10 \mathrm{wt} \%$ $L$-alanine-water mixture. This $L$-alanine concentration was chosen since its pitch was such that the theory predicted the switch in twist angle should occur for a thickness typical of the center of the wedge cell. After cooling into the chiral nematic phase, a slightly different-appearing region began to appear at the thicker end of the cell, and slowly (over hours) grew into the middle part of the cell. This is shown in Fig. 11(a), an image recorded several hours after cooling to the chiral nematic phase. Besides the difference in brightness between the two domains, the larger scratches in the glass due to the rubbing are clearly evident in the left-hand (about $120^{\circ}$ twist) domain. These scratches are not visible in any of the wedge cells with much larger pitch. Just as interesting, the following day the left-hand domain had migrated more toward the thinner part of the wedge cell, but now was also beginning to form along the deepest scratches within the left-hand domain, and the

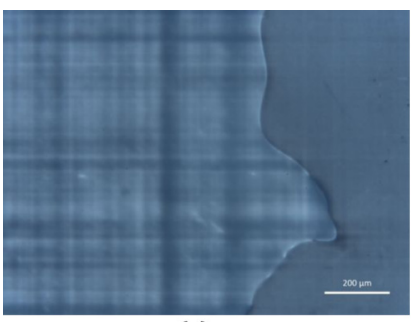

(a)

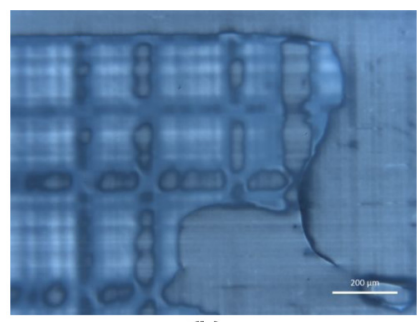

(b)
FIG. 11. (Color online) Domains with very different twist angles in a wedge cell of $15 \mathrm{wt} \%$ DSCG in a $10 \mathrm{wt} \%$ mixture of $L$-alanine and water. The images were taken using a $4 \times$ objective, perpendicular polarizers, and at $25{ }^{\circ} \mathrm{C}$. (a) A domain with a twist angle of slightly more than $240^{\circ}$ growing from the right side (thicker part of the wedge cell) into a domain with a twist angle of slightly less than $120^{\circ}$ several hours after cooling into the chiral nematic phase. (b) The same sample the following day at a thinner part of the cell. Larger scratches from the perpendicular rubbing are clearly visible in both left domains and the right domain of panel (b), but barely visible in the right domain of panel (a). Notice that in panel (b) the $240^{\circ}$ twist domain seems to form first along some of the rubbing scratches in the $120^{\circ}$ twist domain.

scratches were now visible in the right-hand domain. These observations are shown in Fig. 11(b).

\section{DISCUSSION}

Although the nematic phases of thermotropic and chromonic liquid crystals are macroscopically identical and share the same qualitative description of their properties, the values of the various parameters are being measured in chromonics and differences are being revealed. For example, the twist elastic constant in chromonics is smaller than the splay and bend elastic constants by much more than is the case for thermotropic liquid crystals. This difference causes chromonic nematics to have qualitatively different behavior; for example, the twist that spontaneously occurs in achiral chromonic tactoids in the nematic-isotropic biphasic region [24]. Likewise, the anchoring strengths being measured in chromonic liquid crystals are much smaller than have been achieved for thermotropic liquid crystals (for example, $10^{-4} \mathrm{~J} / \mathrm{m}^{2}$ for thermotropics with oblique $\mathrm{SiO}_{2}$ evaporation [19]). Of course, there have been many decades of work on the alignment of thermotropic liquid crystals, so it could be that in the future higher anchoring strengths will be routinely achieved for chromonic liquid crystals. But at this time, both the planar anchoring strengths reported here for DSCG and SSY and the polar anchoring strength estimated for DSCG [9] are $10^{-6} \mathrm{~J} / \mathrm{m}^{2}$ at best, when typical planar anchoring strengths for thermotropic liquid crystals on rubbed glass tend to be in the $10^{-6}$ to $10^{-5} \mathrm{~J} / \mathrm{m}^{2}$ range [19].

The weak anchoring strength results in some other fundamental differences between the behavior of thermotropic and chromonic liquid crystals. For example, the offset angles for these chromonic liquid crystals are much larger than for thermotropic liquid crystals with higher anchoring strengths. One other difference is the stability of twist domains in achiral chromonic samples. The left- and right-handed twist domains in an achiral thermotropic nematic slowly anneal until there is 
only one type of domain present. This is expected because each type of domain has the same elastic free energy associated with it, but there is additional elastic free energy associated with the boundaries between domains. In fact, the twist domains in thermotropic liquid crystals can only be made stable if spacers are dispersed throughout the sample to pin the boundaries between domains and prevent further annealing [25]. For achiral chromonic liquid crystals, the domains are extremely stable without the addition of spacers. The reasons for this undoubtedly stem from the lower twist elastic constant and lower anchoring strength, both of which lower the free energy differences between the domains and the boundaries between them.

Still, the achievement of a simple alignment technique is a big step forward in the study of chromonic liquid crystals. It allows both for further advancement in the scientific understanding of these phases and for the possibility of novel applications. The work reported here, demonstrating that the ability to control the alignment of the chromonic phase allows both the planar anchoring strength in achiral materials and the pitch in chiral materials to be measured, is an example.

The study of chiral nematic chromonic liquid crystals presented here is also a sign of progress since so little has been done on chiral chromonic systems. For example, in one of the few examples of work done in this area, the dependence of the inverse pitch of DSCG on the $L$-alanine concentration was investigated by analyzing fingerprint textures [11]. A linear relationship was found with an HTP of about $0.9 \times 10^{-2} \mu \mathrm{m}^{-1}$ wt $\%^{-1}$, but the trend did not come close to zero inverse pitch at zero $L$-alanine concentration. These measurements have been repeated in the work reported here, and it is clear that the lack of consistent fingerprint textures throughout a chromonic sample hinders the utility of this method of finding the pitch. Only when many measurements on the same sample were averaged did consistent results from samples with different concentrations emerge. This shows that being able to measure the pitch in another way is extremely useful, and one way to do that is to use 90-degree twist cells.

It should also be pointed out that the HTP measured for $L$-alanine in $15 \mathrm{wt} \% \mathrm{DSCG}$ is not unusual for lyotropic systems. For example, different chiral dopants in $28.3 \mathrm{wt} \%$ cetyldimethylethylammonium bromide $2.3 \mathrm{wt} \%$ decanol $67.4 \mathrm{wt} \%$ water produce HTPs in the range of $(0.02$ to 0.4$) \times$ $10^{-2} \mu \mathrm{m}^{-1} \mathrm{wt}^{-1}$ [26]. However, tomatine in a $50: 50$ mixture of cesium pentadecafluoro-octanoate and water has an HTP of $3.3 \times 10^{-2} \mu \mathrm{m}^{-1} \mathrm{wt} \%^{-1}$ [26]. On the other hand, HTPs in thermotropic liquid crystals can be much larger. Some substituted oxiranes and thiiranes in the nematic liquid crystal $p$-methoxybenzylidene $p$ - $n$ butylaniline yield HTPs in the range of $(0.5$ to 40$) \times 10^{-2} \mu \mathrm{m}^{-1} \mathrm{wt} \%^{-1}$ [27]. A chiral isomer of a molecule very similar to DSCG has been synthesized, 5'DSCG-(R,R)-divol, and an $18 \mathrm{wt} \%$ mixture in water has a pitch of about $10 \mu \mathrm{m}$ [12].

There may be an interesting reason why pitch measurements using fingerprint textures are problematic in chromonic liquid crystals. Striped textures have been observed in homeotropic cells of achiral SSY in the nematic phase [23]. but whatever this texture is, it is not stable and disappears after a few minutes. This indicates that an achiral chromonic liquid crystal may twist when subjected to certain homeotropic surface interactions, which is something a thermotropic liquid crystal rarely does. One must therefore ask if the addition of a chiral dopant might make a fingerprint texture that does not accurately reflect the intrinsic chirality stable in some situations. If this is the case, use of the fingerprint texture to measure the pitch in chromonic liquid crystals may be problematic.

In order to verify that the two domains shown in Fig. 11 are in fact due to a change from a twist of about $120^{\circ}$ to a twist of around $240^{\circ}$, the optical transmission was theoretically calculated for both twist angles using the parameters measured for the $15 \mathrm{wt} \%$ DSCG solutions. For both parallel and perpendicular polarizers, the theory predicted that the maximum and minimum transmission as the angle of the sample is varied should be similar for the two domains, but that for parallel polarizers the domain with about a $240^{\circ}$ twist should have higher values of $I_{C}$ and $I_{S}$, while for perpendicular polarizers the approximately $120^{\circ}$ twist domain should have higher values of $I_{C}$ and $I_{S}$. Measurements of the optical transmission of the two domains verified this assuming the domain on the thinner side of the wedge cell has a twist angle of around $120^{\circ}$ and the domain on the thicker side of the wedge cell has a twist angle of around $240^{\circ}$.

It is also interesting to speculate why the larger scratches are observable in these domains and not in any of the other samples where the twist angle is much less than $120^{\circ}$. While the measurements of the anchoring strength reported here clearly represent an average stemming from a distribution of scratch size, there is evidence that the anchoring strength varies with scratches of different depths and widths [17]. If the offset angle is large, local differences in orientation due to varying scratch depth and width are most likely to be large and thereby show a variation in optical transmission correlated with the scratches. This local variation in the anchoring strength may even be enough to force the change from twist angles of slightly less than $120^{\circ}$ to slightly more than $240^{\circ}$ only in certain locations, as seems to be the case in Fig. 11(b). Furthermore, perhaps the offset angle in the right-hand domain of Fig. 11(a) is not very large, so the variation in transmission is barely evident, but over time and in a different part of the wedge cell, perhaps the offset angle in the right-hand domain is large enough for the local variations to be seen, as in Fig. 11(b).

\section{CONCLUSIONS}

Simple rubbing of glass surfaces with a very fine abrasive achieves planar alignment in the nematic phase of at least two chromonic liquid crystals. This ability allows the chromonic liquid crystal in its nematic phase to be distorted by arranging the rubbing directions of the top and bottom pieces of glass to be at $90^{\circ}$ to one another. The anchoring of the director to the substrate is weak, causing large offset angles to occur. Such a chromonic sample, whether nematic or chiral nematic, can be examined optically and fundamental parameters such as birefringence, planar anchoring strength, and chiral pitch measured. For $15 \mathrm{wt} \%$ DSCG and $30 \mathrm{wt} \% \mathrm{SSY}$, the planar anchoring strength measured using this rubbing technique is quite weak, about $4 \times 10^{-7} \mathrm{~J} / \mathrm{m}^{2}$. The helical twisting power of $L$-alanine in $15 \mathrm{wt} \%$ DSCG turns out to be about $1 \times$ $10^{-2} \mu \mathrm{m}^{-1} \mathrm{wt} \%^{-1}$, which is comparable to what is measured for some lyotropic systems, but less than is typically observed for thermotropic liquid crystals. 


\section{ACKNOWLEDGMENTS}

Acknowledgment is made to the National Science Foundation for financial support through Grant No. UPENN MRSEC DMR-1120901. C.K.M. acknowledges support from the Research Experiences for Undergraduates Program at the Laboratory for Research in the Structure of Matter at

[1] J. Lydon, Liq. Cryst. 38, 1663 (2011).

[2] S. W. Tam-Chang and L. Huang, Chem. Commun. 2008, 1957 (2008).

[3] H. S. Park and O. D. Lavrentovich, in Liquid Crystals Beyond Displays: Chemistry, Physics, and Applications, edited by Q. Li (John Wiley and Sons, New York, 2012), Chap. 14, pp. 449-483.

[4] S. V. Shiyanovskii, T. Schneider, I. I. Smalyukh, T. Ishikawa, G. D. Niehaus, K. J. Doane, C. J. Woolverton, and O. D. Lavrentovich, Phys. Rev. E. 71, 020702 (2005).

[5] C. J. Woolverton, E. Gustely, L. Li, and O. D. Lavrentovich, Liq. Cryst. 32, 417 (2005).

[6] V. R. Horowitz, L. A. Janowitz, A. L. Modic, P. A. Heiney, and P. J. Collings, Phys. Rev. E 72, 041710 (2005).

[7] Y. A. Nastishin, H. Liu, T. Schneider, V. Nazarenko, R. Vasyuta, S. V. Shiyanovskii, and O. D. Lavrentovich, Phys. Rev. E 72, 041711 (2005).

[8] Yu. A. Nastishin, K. Neupane, A. R. Baldwin, O. D. Lavrentovich, and S. Sprunt, Electronic Liquid Crystal Communications, July 15 (2008).

[9] V. G. Nazarenko, O. P. Boiko, H. S. Park, O. M. Brodyn, M. M. Omelchenko, L. Tortora, Y. A. Nastishin, and O. D. Lavrentovich, Phys. Rev. Lett 105, 017801 (2010).

[10] S. Zhou, Y. A. Nastishin, M. M. Omelchenko, L. Tortora, V. G. Nazarenko, O. P. Boiko, T. Ostapenko, T. Hu, C. C. Almasan, S. N. Sprunt, J. T. Gleeson, and O. D. Lavrentovich, Phys. Rev. Lett 109, 037801 (2012).

[11] H. Lee and M. M. Labes, Mol. Cryst. Liq. Cryst. 84, 137 (1982). the University of Pennsylvania. P.J.C. acknowledges support from the Alexander von Humboldt Foundation for his visit to the University of Paderborn. N.Z. and H.-S.K. acknowledge support from the German Research Foundation (DFG, GRK 1464). The parylene-coated slides were kindly provided by Joonwoo Jeong at the University of Pennsylvania.
[12] S. Yang, B. Wang, D. Cui, D. Kerwood, S. Wilkens, J. Han, and Y. Y. Luk, J. Phys. Chem. B 117, 7133 (2013).

[13] K. Ichimura, T. Fujiwara, M. Momose, and D. Matsunaga, J. Mater. Chem. 12, 3380 (2002).

[14] T. Fujiwara and K. Ichimura, J. Mater. Chem. 12, 3387 (2002).

[15] C. M. Tone, M. P. D. Santo, M. G. Buonomenna, G. Golemme, and F. Ciuchi, Soft Matter 8, 8478 (2012).

[16] H. S. Jeong, H. J. Jeon, Y. H. Kim, M. B. Oh, P. Kumar, S. W. Kang, and H. T. Jung, NPG Asia Mater. 4, e7 (2012).

[17] M. Srinivasarao (private communication) (2013).

[18] V. P. Vorflusev, H. S. Kitzerow, and V. G. Chigrinov, Jpn. J. Appl. Phys. 34, L1137 (1995).

[19] L. M. Blinov and V. G. Chigrinov, Electrooptic Effects in Liquid Crystal Materials (Springer-Verlag, New York, 1993) pp. 118-120.

[20] L. M. Blinov, Structure and Properties of Liquid Crystals (Springer, Heidelberg, 2012) pp. 199-205, 274-275.

[21] E. P. Raynes, Liq. Cryst. 34, 697 (2007).

[22] P. McIntyre, J. Opt. Soc. Am. 68, 869 (1978).

[23] J. Jeong (private communication) (2003).

[24] L. Tortora and O. D. Lavrentovich, Proc. Natl. Acad. Sci. USA 108, 5163 (2011).

[25] E. P. Raynes, Liq. Cryst. 33, 1215 (2006).

[26] E. Figgemeier and K. Hiltrop, Liq. Cryst. 26, 1301 (1999).

[27] G. Gottarelli, P. Mariani, G. P. Spada, B. Samori, A. Forni, G. Solladie, and M. Hibert, Tetrahedron 39, 1337 (1983). 